\title{
Atividade física e hábitos alimentares de adolescentes de três escolas públicas de Florianópolis/sc
}

\author{
Physical activity and nutritional habits of teenagers from three \\ public schools in Florianópolis/sC
}

\author{
Eliane Denise da Silveira Araújo ${ }^{1}$ \\ Nelson Blank 2 \\ Walter Ferreira de Oliveira ${ }^{2}$
}

1 Universidade Estadual
de Ponta Grossa. Departamento de Educação Física. Ponta Grossa, PR, Brasil.

2 Universidade Federal de Santa Catarina. Departamento de Saúde Pública. Florianópolis, SC. Brasil.

Recebido em 03/05/08 Revisado em 13/07/08 Aprovado em 03/03/09
Resumo - Investigar hábitos alimentares e de atividade física de adolescentes de 16 e 17 anos, de três escolas públicas de Florianópolis/SC. A amostra foi composta por 720 escolares, sendo 252 rapazes e 468 moças. Foram aplicados dois questionários enviados a estudantes e seus pais. $\mathrm{Na}$ análise dos dados, utilizaram-se os recursos da estatística descritiva (média e desvio padrão) e para análise de diferenças entre grupos, utilizou-se o teste do qui-quadrado com intervalo de confiança de 95\%. Cerca de 69,1\% dos adolescentes praticavam Educação Física regular na escola, mas 59,6\% não faziam atividade física organizada fora dela. Do total de adolescentes $(40,4 \%)$ que praticavam atividade física organizada fora da escola, 55,9\% são do sexo masculino e $35,1 \%$ do sexo feminino; a maioria $(61,6 \%)$ praticava atividade física de uma a três vezes por semana. Com relação ao nível de atividade física, a maioria dos escolares (48,3\%) foi considerada na categoria: "intermediários ativos" e 30,6\% como "ativos", sendo os rapazes em maior proporção $(43,6 \%)$ que as moças $(23,5 \%)$. Verificou-se que $54,5 \%$ dos escolares assistiam TV acima de três horas/dia. Quanto aos hábitos alimentares, cerca de 55,4\% apresentavam uma alimentação considerada saudável, segundo os parâmetros utilizados pelos autores, sendo os rapazes $(61,5 \%)$ em maior proporção do que as moças $(51,9 \%)$. Os rapazes consumiam mais leite que as moças, enquanto estas indicaram um maior consumo de doces do que os rapazes. De modo geral, observou-se que a grande maioria dos adolescentes apresentou comportamentos de saúde positivos, como bons hábitos de atividade física e alimentação.

Palavras-chave: Saúde do adolescente; Hábitos alimentares; Atividade motora.

Abstract - The objective of this study was to investigate the dietary habits and physical activity of adolescents aged 16 to 17 years from public schools in Florianópolis, SC. The sample consisted of 720 students, including 252 boys and 468 girls. Two questionnaires were applied, which were sent to the students and their parents. Descriptive statistics (mean and standard deviation) was used for data analysis and differences between groups were analyzed by the chi-square test, with a 95\% confidence interval. Among the adolescents studied, $69.1 \%$ participated regularly in physical education at school, whereas $59.6 \%$ did not perform any physical activity outside of school. Among the adolescents who performed organized physical activity outside of school (40.4\%), 55.9\% were boys and 35.1\% were girls, and most of them (61.6\%) performed physical activity three times per week. With respect to physical activity level, most students (48.3\%) were classified as active at the intermediate level and 30.6\% were classified as active, with a higher proportion of boys (43.6\%) than girls (23.5\%). In addition, 54.5\% of the students watched TV for more than 3 h/day. Regarding dietary habits, 55.4\% of the adolescents presented a healthy diet according to the parameters used in this study, with a higher proportion of boys (61.5\%) than girls $(51.9 \%)$. Boys consumed more milk than girls who, in turn, reported a higher consumption of candy compared to boys. In general, most of the adolescents presented positive health behaviors such as good physical activity and dietary habits.

Key words: Adolescent health; Food habits; Motor activity. 


\section{INTRODUÇÃO}

$\mathrm{Na}$ adolescência, a influência da família, dos amigos e de todo o contexto socioeconômico e cultural no qual o adolescente se insere é primordial em suas decisões cotidianas, fazendo do adolescente um ser eminentemente ligado a grupos, contextualizados em diversas culturas e subculturas. Assim, o ambiente social e o contexto econômico-cultural contribuem muito para orientações dos hábitos alimentares e de atividade física dos adolescentes ${ }^{1}$.

Muitos autores sustentam que os adolescentes têm, em geral, maus hábitos alimentares. Triches e Giugliani ${ }^{2}$ argumentam que a alimentação atual é baseada no insuficiente consumo de frutas, hortaliças e leite, no aumento do consumo de guloseimas (bolachas recheadas, salgadinhos e doces) e refrigerantes, bem como na omissão do café da manhã, o que contribui para adoção de hábitos alimentares negativos e consequentemente, para o surgimento de diversas doenças crônicas como obesidade, diabetes e hipertensão.

Tem sido também apontada a crescente tendência dos adolescentes à inatividade física ${ }^{3-5,8}$. A sociedade contemporânea, segundo estes autores, embora cada vez mais agitada e competitiva, oferece comodidades que favorecem a não realização de esforços físicos, tornando o ser humano cada vez mais sedentário, com diversas consequências à sua saúde .

A saúde dos adolescentes, contextualizada a partir dos riscos trazidos pelo sedentarismo e pela adoção de hábitos alimentares potencialmente danosos, torna-se um componente da esfera da Saúde Pública, tendo sua importância ampliada por ser esta fase um período estratégico em relação às escolhas que caracterizam estilos de vida que podem repercutir ou se perpetuar na idade adulta ${ }^{1}$.

Além disso, estudos com adolescentes nas escolas, podem servir como mecanismo de identificação, monitoração e vigilância de comportamentos de risco à saúde, prevenindo possíveis agravos à saúde em âmbito escolar, extensivos a toda população desta faixa etária.

Desta forma, considerando que esta fase do ciclo da vida é crucial para a adoção de hábitos, atitudes e comportamentos que condicionam estilos de vida que podem repercutir na idade adulta, conduziu-se este estudo com objetivo de descrever e analisar hábitos alimentares e de atividade física, de adolescentes de 16 e 17 anos, de ambos os sexos, de três escolas públicas de Florianópolis, SC.

\section{PROCEDIMENTOS METODOLÓGICOS}

Trata-se de um estudo transversal, com coleta de dados durante o ano escolar de 2005, no município de Florianópolis. A população do estudo foi composta por 1963 escolares, de ambos os sexos, na faixa etária de 16 e 17 anos, matriculados em três escolas públicas, do ensino médio, da região central da cidade de Florianópolis. A faixa etária foi escolhida dada à semelhança de hábitos proporcionada pela proximidade das idades e possível aumento de fatores de confusão com inclusão de jovens de outras idades. As escolas foram selecionadas intencionalmente, pelo fato de serem as maiores do município e localizadas na região central do mesmo.

O tamanho da amostra foi calculado para uma prevalência dos vários componentes do estilo de vida de 50\%, com uma margem de erro de 5\%, e um nível de confiança de $95 \%$. O "n" mínimo calculado para o estudo ser representativo das escolas foi de 637 adolescentes. Todos os alunos das escolas selecionadas foram convidados a participar voluntariamente da pesquisa. Participou do estudo um total de 720 escolares.

Os dois questionários utilizados para a coleta de dados eram autoadministráveis e entregues aos alunos. Um deles, dirigido aos pais, versava sobre características sóciodemográficas e o outro, sobre estilo de vida, para serem respondidos pelos estudantes e devolvidos no dia seguinte. Caso esquecessem de responder, os pesquisadores retornavam nos dias posteriores até recolher o máximo possível de questionários. Para realizar a coleta, os alunos levavam para casa, também, o termo de consentimento livre e esclarecido para os pais assinarem, autorizando-os a participarem na pesquisa.

Utilizaram-se as seguintes variáveis no estudo: características sóciodemográficas (sexo, trabalho remunerado, co-habitação, estado civil dos pais e grau de escolaridade destes, delimitado pelos anos referidos de estudo); atividade física (Educação Física regular, horas semanais de atividade física organizada, horas de TV e computador e nível de atividade física, por categoria previamente estipulada); e alimentação saudável ou não saudável, inferida a partir de uma lista de hábitos alimentares (tomar café da manhã, consumo de leite e ou yogurte, frutas, vitaminas ou suco de fruta natural, verduras, lanches gordos, doces, sorvete e chocolate). Elaborou-se, para fins de estabelecer esta variável, uma escala, com escore de 0 (zero) a dez, através do somatório das variáveis: café, leite, fruta, verdura, gordura e doce; e tomou-se como ponto de corte 
a mediana ${ }^{4}$. $\mathrm{O}$ escore de 0 (zero) a 4 (quatro) foi considerado alimentação saudável e o de 5 (cinco) a 10 (dez) alimentação não saudável.

Para fins deste estudo, considerou-se atividade física organizada a que se realiza em academia, centros esportivos e outros estabelecimentos do gênero, e supervisionada por profissionais de Educação Física.

O nível de atividade física foi classificado em: "ativo" (aqueles que praticavam Educação Física regular na escola e faziam atividade física organizada fora da escola); "intermediário ativo" (aqueles que faziam Educação Física regular e não faziam atividade física organizada; ou o contrário: aqueles que não faziam Educação Física regular na escola, mas faziam atividade física organizada fora da escola) e "não ativo" (aqueles que não faziam Educação Física na escola ou fora dela).

Para a análise de diferenças entre grupos, utilizou-se o teste do qui-quadrado. Considerou-se como estatisticamente significante aquelas diferenças com um valor de $\mathrm{p} \leq 0,05$.

O projeto foi previamente submetido e aprovado pelo Comitê de Ética da Universidade Federal de Santa Catarina (Projeto n. 125/04). No banco de dados, não houve personalização dos registros de cada um dos entrevistados.

\section{RESULTADOS}

Participaram do estudo 720 escolares, sendo 252 rapazes (35\%) e 468 moças (65\%). A maioria dos adolescentes não exercia trabalho remunerado, tinha pais casados, moravam com ambos os pais, e tinham pai e mãe com 11 anos ou mais de escolaridade.

Na tabela 1, são apresentados os hábitos de atividade física dos escolares referentes à prática de Educação Física regular, prática de atividade física organizada fora da escola, atividade física organizada por semana, horas de televisão por dia e nível de atividade física.

A maioria (69,1\%) dos adolescentes estudados praticava Educação Física regular na escola, não havendo diferenças entre os sexos, mas não fazia atividade física organizada fora dela (59,6\%). Dos que praticavam atividade física fora da escola, os rapazes eram em maior proporção $(55,95 \%)$. A maioria $(61,6 \%)$ dos escolares praticava atividade física organizada fora da escola, de uma a três vezes por semana.

A maioria dos adolescentes (54,5\%) assistia TV e ou usava computador por mais de três horas diárias, sendo classificada como intermediário ativo $(48,3 \%)$. No entanto, em sua cotidianidade, os rapazes $(43,65 \%)$ eram mais ativos que as moças.

A tabela 2 apresenta uma descrição dos hábitos alimentares dos escolares, referente ao hábito de tomar café da manhã (desjejum), números de copos de leite e ou yogurte ingeridos diariamente, consumo de frutas, vitaminas ou sucos de frutas naturais, verduras (ex. alface, rúcula, agrião, etc.) e legumes (ex. tomate, cenoura, abobrinha, etc.),

Tabela 1. Hábitos de atividade física dos escolares de 16 e 17 anos de ambos os sexos de Florianópolis.

\begin{tabular}{|c|c|c|c|c|c|c|c|}
\hline \multirow[t]{2}{*}{ Variável } & \multicolumn{2}{|c|}{ Total } & \multicolumn{2}{|c|}{ Masculino } & \multicolumn{2}{|c|}{ Feminino } & \multirow[t]{2}{*}{$p$} \\
\hline & $\%$ & $\mathrm{n}$ & $\%$ & $n$ & $\%$ & $\mathrm{n}$ & \\
\hline \multicolumn{8}{|l|}{ Educação Física regular } \\
\hline Sim & 69,1 & 498 & 72,62 & 183 & 67,31 & 315 & ns \\
\hline Não & 30,9 & 223 & 27,38 & 69 & 32,69 & 153 & \\
\hline \multicolumn{8}{|c|}{ Atividade física organizada } \\
\hline Sim & 40,4 & 291 & 55,95 & 141 & 32,12 & 150 & $<0,001$ \\
\hline Não & 59,6 & 429 & 44,05 & 111 & 67,88 & 317 & \\
\hline \multicolumn{8}{|c|}{ Atividade física organizada por semana } \\
\hline$>3$ vezes/semana & 38,4 & 113 & 42,96 & 61 & 34,21 & 52 & ns \\
\hline 1 a 3 vezes/semana & 61,6 & 181 & 57,04 & 81 & 65,79 & 100 & \\
\hline \multicolumn{8}{|c|}{ Nível de atividade física } \\
\hline Ativo & 30,6 & 220 & 43,65 & 110 & 23,55 & 110 & 0,001 \\
\hline Intermediário ativo & 48,3 & 348 & 41,27 & 104 & 52,25 & 244 & \\
\hline Inativo & 21,1 & 152 & 15,08 & 38 & 24,20 & 113 & \\
\hline \multicolumn{8}{|l|}{ Horas de televisão } \\
\hline Até 2 hs/dia & 45,5 & 327 & 40,80 & 102 & 47,97 & 224 & ns \\
\hline 3 a 4 hs/dia & 28,3 & 203 & 28,40 & 71 & 28,27 & 132 & \\
\hline$>4$ horas/dia & 26,2 & 188 & 30,80 & 77 & 23,77 & 111 & \\
\hline
\end{tabular}


Tabela 2. Hábitos alimentares dos escolares de 16 e 17 anos de ambos os sexos de Florianópolis.

\begin{tabular}{|c|c|c|c|c|c|c|c|}
\hline \multirow[t]{2}{*}{ Variável } & \multicolumn{2}{|c|}{ Total } & \multicolumn{2}{|c|}{ Masculino } & \multicolumn{2}{|c|}{ Feminino } & \multirow[t]{2}{*}{$\mathrm{p}$} \\
\hline & $\%$ & $\mathrm{n}$ & $\%$ & $\mathrm{n}$ & $\%$ & $n$ & \\
\hline \multicolumn{8}{|l|}{ Café da manhã } \\
\hline Sim & 64,6 & 465 & 60,71 & 153 & 66,81 & 312 & ns \\
\hline Não & 35,4 & 255 & 39,29 & 99 & 33,19 & 155 & \\
\hline \multicolumn{8}{|c|}{ № de copos de leite } \\
\hline$>2$ copos/dia & 9,5 & 68 & 13,89 & 35 & 7,08 & 33 & 0,009 \\
\hline 1 a 2 copos/dia & 62,9 & 452 & 61,51 & 155 & 63,52 & 296 & \\
\hline Nenhum & 27,7 & 199 & 24,60 & 62 & 29,40 & 137 & \\
\hline \multicolumn{8}{|l|}{ Fruta } \\
\hline Sim & 56,8 & 409 & 59,13 & 149 & 55,46 & 259 & ns \\
\hline Não & 43,2 & 311 & 40,87 & 103 & 44,54 & 208 & \\
\hline \multicolumn{8}{|l|}{ Verduras } \\
\hline$>1 x /$ dia & 10,7 & 77 & 9,92 & 25 & 11,13 & 52 & ns \\
\hline Às vezes/ 1x/dia & 77,8 & 560 & 76,98 & 194 & 78,16 & 365 & \\
\hline Nunca & 11,5 & 83 & 13,10 & 33 & 10,71 & 50 & \\
\hline \multicolumn{8}{|l|}{ Doces } \\
\hline Até 1x/sem & 46,5 & 335 & 58,33 & 147 & 40,04 & 187 & 0,001 \\
\hline 2 a 3 x/sem & 34,7 & 250 & 30,56 & 77 & 37,04 & 173 & \\
\hline$>3 \times /$ sem & 18,8 & 135 & 11,11 & 28 & 22,91 & 107 & \\
\hline \multicolumn{8}{|l|}{ Lanches gordos } \\
\hline Até $1 x /$ sem & 51,1 & 368 & 49,60 & 125 & 51,82 & 242 & ns \\
\hline 2 a 3 x/sem & 34,7 & 250 & 34,52 & 87 & 34,90 & 163 & \\
\hline$>3 \times /$ sem & 14,2 & 102 & 15,87 & 40 & 13,28 & 62 & \\
\hline \multicolumn{8}{|c|}{ Alimentação saudável } \\
\hline saudável & 55,4 & 398 & 61,51 & 155 & 51,93 & 242 & 0,015 \\
\hline não-saudável & 44,6 & 321 & 38,49 & 97 & 48,07 & 224 & \\
\hline
\end{tabular}

lanches gordos como pizzas, hambúrgueres, empanadas, esfihas, pastéis, cachorros-quentes e chips; e doces, sorvetes e chocolate.

A partir destes hábitos, os autores categorizaram os adolescentes como tendo ou não uma alimentação saudável. Por exemplo, são considerados como tendo uma alimentação mais saudável os adolescentes que têm o hábito do desjejum, que consomem diariamente frutas, verduras (pelo menos uma vez ao dia), leite (pelo menos dois copos ao dia), e que ingerem gorduras e doces somente até uma vez por semana.

Observa-se que a maioria dos escolares (55,4\%) apresentava uma alimentação considerada saudável, sendo os rapazes em maior proporção $(61,5 \%)$ do que as moças. Os rapazes ingeriam alimentos com maiores fontes de proteínas e cálcio como leite, em contraposição às moças que ingeriam mais frequentemente alimentos menos nutritivos e com maiores teores de calorias, como doces.

\section{DISCUSSÃO}

O adolescente, atualmente, tende há permanecer muito tempo sentado na frente da TV ou computador, reservando pouco tempo a atividades práticas. Os resultados encontrados neste trabalho estão de acordo com os valores encontrados na literatura. Nesta amostra, a maioria dos escolares (54,5\%), tanto do sexo masculino quanto do feminino, vê TV por mais de três horas por dia, enquanto $45,5 \%$ assistem TV até duas horas por dia. Estudos ${ }^{7-9}$ na literatura apontam valores semelhantes, nos quais rapazes e moças vêem TV, em média, de 3,2 horas a 4,9 horas por dia.

Sabe-se da importância da televisão como forma de lazer na sociedade contemporânea. Esta magnitude se amplia, principalmente, entre indivíduos de nível socioeconômico mais baixo, os quais não têm acesso a muitas opções de lazer e optam pela TV como forma primordial de distração e entretenimento. Associa-se a isto o fato de as atividades 
de assistir TV e ou tempo de computador proporcionarem maior segurança aos pais, que preferem ver seus filhos em casa a expô-los aos problemas identificados nas ruas das grandes cidades ${ }^{10}$.

Aspectos importantes podem ser associados ao número de horas diárias que o adolescente gasta na frente da TV, como: os modismos impostos pela TV que podem influenciar na escolha de determinado produto, favorecendo a aquisição de hábitos alimentares inadequados ${ }^{9,11}$ e o sedentarismo. Ou seja, o tempo de exposição à TV e ao computador pode, neste sentido, servir como indicador de vida sedentária e, consequentemente, contribuir para o sobrepeso e a obesidade?

Alves ${ }^{12}$ sustenta que um adolescente assiste, em média, a 27 horas de programas de TV por semana. Somando a isso o tempo do uso de computadores e o tempo que o adolescente é obrigado a ficar sentado na escola, sobra relativamente pouco tempo para atividades físicas. Talvez o tempo de permanência do adolescente em frente à televisão e ou ao computador poderia ser ocupado por atividades físicas lúdicas, que exijam maior gasto energético, o que poderia contribuir para uma vida mais saudável e com maior qualidade.

Neste estudo, a maioria dos escolares $(69,1 \%)$ pratica Educação Física regular na escola, o que confirma que a disciplina de Educação Física na escola, embora desvalorizada e perdendo seu espaço, continua sendo bastante praticada pelos adolescentes. A Educação Física é, ao mesmo tempo, uma opção de lazer e de prática de atividades físicas, sendo crucial, sobretudo, para pessoas que não podem pagar para frequentar clubes ou academias, pois é aberta a todos os alunos, independente de condições socioeconômicas.

No que se refere à prática de atividade física organizada, este estudo corrobora os estudos de França et al. ${ }^{9}$ e Matos et al. ${ }^{13}$, os quais mostram que cerca de $47 \%$ a $53 \%$ dos entrevistados praticavam de uma a três horas diárias de atividade física fora da escola.

Com relação à influência do gênero, este estudo corrobora os trabalhos encontrados na literatura $3,4,7,8,14,15$, os quais apontam que os rapazes são mais ativos que as moças em sua cotidianidade. O estudo foi também semelhante aos dados de prevalência encontrados no trabalho de Matsudo et $\mathrm{al}^{4}{ }^{4}, \mathrm{com}$ adolescentes paulistas, nos quais os "ativos" apresentaram uma prevalência de 45,8\%, os "irregularmente ativos" de $36 \%$ e os "sedentários" ou "inativos" de 7,7\%.

Comparando com outros estudos, verificou-se que são poucos os adolescentes inativos em Floria- nópolis (21,1\%), em comparação aos adolescentes pesquisados em outros estudos ${ }^{3,5,7,8,15,16}$ que apresentam maiores valores de inatividade (de 39\% a $80 \%)$. O fato de haver menos indivíduos inativos pode ser justificado pela seleção das escolas, que são frequentadas por alunos de nível socioeconômico mais elevado, além de pais com melhor nível de instrução, favorecendo a busca por atividade física organizada fora da escola e a prática de lazer ativo. Para Oehlschlaeger et al. ${ }^{15}$,o nível socioeconômico baixo está diretamente relacionado ao sedentarismo e a pouca atividade física. Consequentemente, indivíduos de classes econômicas mais favorecidas tendem a manter maiores cuidados com sua saúde, neste sentido, com maior índice de práticas habituais de atividade física.

Com relação aos hábitos alimentares, sabe-se que, geralmente, nesta fase os adolescentes passam a maior parte do tempo fora de casa, na escola e com os amigos, o que pode influenciar na aquisição de uma alimentação rica em lanches e fast foods entre as refeições. Esta atitude pode ser justificada pela falta de tempo disponível para dedicar a uma refeição, por preferências individuais, por modismo ou por ser uma refeição que pode ser feita com os amigos ${ }^{9}$.

Neste estudo, os rapazes $(13,9 \%)$ tendem a ingerir mais de dois copos de leite por dia, enquanto que as moças $(22,9 \%)$ ingerem doces mais de três vezes por semana. Assim, os dados encontrados neste estudo corroboram os estudos de Kazapi et al..$^{17}$ que apontam que os rapazes tendem a consumir mais carboidratos e proteínas e menos lipídios e doces, comportando-se as moças exatamente ao contrário.

Pode-se observar, também, que a ingestão de doces e lanches gordos, acima de três vezes por semana, na amostra como um todo apresentou uma frequência relativamente baixa $(18,8 \%$ e 14,2\% respectivamente), em comparação com a literatura que aponta valores de aproximadamente $60 \%$ a $70 \%$ para a ingestão de doces ${ }^{3,9,13,18}$ e lanches gordos ${ }^{5,9,18}$. Com exceção do estudo de Farias Junior ${ }^{3}$,no qual o consumo de frituras em mais de quatro dias por semana foi de 12 a 20\% entre os adolescentes pesquisados.

Com relação ao consumo de leite e verduras, apenas 9,5\% dos escolares ingerem mais de dois copos de leite por dia e 10,7\% consomem verduras e legumes mais de uma vez ao dia. Esta frequência de consumo pelos adolescentes pesquisados neste estudo não pode ser vista como ponto positivo, se consideramos estes alimentos como componentes nutricionais necessários à alimentação do adolescente.

Comparando a ingestão de leite com a literatura, os resultados obtidos neste estudo estão abaixo 
dos valores encontrados nos estudos de Farias Júnior ${ }^{(3)}$ e Santos et al. ${ }^{17}$, nos quais a proporção de adolescentes que referiu ingerir leite é de $36,7 \%$ a 91,3\%. No entanto, semelhante ao estudo de França et al. ${ }^{9}$ no qual $11 \%$ dos adolescentes consumem diariamente leite e derivados.

No que concerne aos vegetais, a literatura aponta valores também mais elevados. Em diferentes estudos foram encontrados valores de 25 a $40 \%$ para os adolescentes que apresentaram hábito de consumir vegetais diariamente ${ }^{3,13,18}$.

Neste estudo, 64,6\% dos escolares relataram o hábito de ingerir desjejum não havendo diferenças entre os sexos. Comparando com a literatura, apenas $16 \%$ e $18,1 \%$ dos escolares disseram ingerir desjejum diariamente ${ }^{9,17,18}$. No entanto, são frequentes os adolescentes consumirem refeições de modo irregular, tendendo a pular as refeições, principalmente, o desjejum. Isso é mais comum entre as meninas como forma de perder peso, apresentando assim uma dieta inadequada em relação a vários nutrientes.

Analisando o consumo de frutas, neste estudo, 56,8\% dos adolescentes têm hábito de consumir frutas diariamente. Ponto positivo, tendo em vista que foram encontrados na literatura valores inferiores a estes, cerca de $20 \%$ a 30\% 3,5 . Contudo, no estudo de Pires $^{14}$, os resultados indicam que cerca de $85,8 \%$ dos escolares referiram consumir frutas diariamente.

Em virtude das diferentes formas de obtenção e classificação dos resultados, a comparação com outros estudos torna-se dificultada, principalmente, no que concerne aos hábitos alimentares. Esta dificuldade se deve, sobretudo, à ausência de padronização em relação às quantidades de alimentos consumidos. Contudo, pode-se argumentar que, de maneira geral, os hábitos dos adolescentes estudados tendem a ser considerados como saudáveis, em virtude do consumo relativamente baixo de alimentos calóricos e gordurosos como doces e lanches gordos. A proporção de adolescentes que relatou ingestão de frutas e hábito de ingerir desjejum diariamente, sustenta o argumento de que estes adolescentes tendem a uma alimentação mais balanceada e condizente com as necessidades do ser humano.

No entanto, tomando-se em consideração todas as variáveis, quase a metade dos adolescentes $(44,6 \%)$ não apresenta uma alimentação classificada como saudável por estes autores, sendo as moças em maior proporção, dada, no caso destas últimas, a grande quantidade de doces ingeridos.

Para finalizar, algumas limitações do estudo devem ser ressaltadas. Por exemplo, o estudo apre- sentou uma sobre representação sem significação estatística das adolescentes da amostra (468 moças para 252 rapazes). Um dos motivos para isso pode estar relacionado ao fato de as moças apresentarem menor resistência para responderem ao questionário por apresentarem hábitos de vida mais saudáveis. Entretanto, os resultados encontrados e confrontados com a literatura sugerem que, se isto ocorreu, não foi causa de importante viés. Além disso, a validade externa do estudo fica limitada devido à dificuldade em extrapolar estes dados a todos os estudantes de 16 e 17 anos de Florianópolis, perante as barreiras encontradas em pesquisas com escolares que impossibilitaram a realização do estudo de forma aleatória.

\section{CONCLUSÃO}

De maneira geral, este estudo demonstrou que a maioria dos adolescentes pratica Educação Física regular sendo classificada como "intermediários ativos" e apresenta uma alimentação considerada saudável. Com relação a diferenças entre sexos, os rapazes são mais ativos e apresentam hábitos alimentares que são considerados mais saudáveis do que os das moças.

Pode-se observar, também, que a grande maioria dos adolescentes pesquisados apresenta comportamentos de saúde em geral positivos, como bom nível de atividade física e hábitos alimentares saudáveis, não muito diferente do que é relatado na literatura.

É importante chamar a atenção para a necessidade de mais estudos sobre estilo de vida de adolescentes como forma de reforçar a vigilância da saúde e com objetivo de localizar, evitar e, quando necessário, corrigir hábitos negativos de saúde tanto no presente quanto no futuro. Sabe-se que indivíduos que apresentam um estilo de vida não saudável na adolescência podem manter ou piorar os comportamentos de saúde na idade adulta, o que pode trazer diversas consequências como piora dos níveis de sedentarismo, obesidade e alterações crônico-degenerativas na população.

Tendo em vista estas considerações, estes autores defendem, além da necessidade de mais pesquisas nesta área, programas de orientação alimentar e maior ênfase na promoção de atividade física na escola. $\mathrm{O}$ avanço do conhecimento nesta área,pode servir ao propósito de estimular práticas saudáveis de alimentação e atividade física bem como à conscientização de sua importância no crescimento e desenvolvimento integral do adolescente. 


\section{REFERÊNCIAS BIBLIOGRÁFICAS}

1. Ferreira MA, Alvim NAT, Teixeira MLO, Veloso RC. Saberes de adolescentes: estilo de vida e cuidado à saúde. Texto Contexto - Enferm 2007;16(2):217-224.

2. Triches RM, Giugliani ERJ. Obesidade, práticas alimentares e conhecimentos de nutrição em escolares. Rev Saúde Pública 2005;39(4):541-547.

3. Farias Júnior JC. Estilo de vida de escolares do ensino médio no município de Florianópolis. [Dissertação de Mestrado - Programa de Pós Graduação em Educação Física]. Florianópolis (SC): Universidade Federal de Santa Catarina; 2002.

4. Matsudo SM, Matsudo VR, Araújo T, Andrade D, Andrade E, Oliveira L et al. Nível de atividade física da população do Estado de São Paulo: análise de acordo com o gênero, idade, nível socioeconômico, distribuição geográfica e de conhecimento. Rev Bras Ciên Mov 2002;10(4):41-50.

5. Novaes AJ. Estilo de vida relacionado à saúde e hábitos comportamentais em escolares do ensino médio do município de Blumenau/SC: a influência da escola. [Dissertação de Mestrado - Programa de Pós Graduação em Educação Física]. Florianópolis (SC): Universidade Federal de Santa Catarina; 2002.

6. Mendonça CP, Anjos LA. Aspectos das práticas alimentares e da atividade física como determinantes do crescimento do sobrepeso/obesidade no Brasil. Cad Saúde Pública 2004;20(3):698-709.

7. Silva RCR, Malina RM. Nível de atividade física em adolescentes do município de Niterói, Rio de Janeiro, Brasil. Cad Saúde Pública 2000;16(4):1091-1097.

8. Guedes DP, Guedes JERP, Barbosa DS, Oliveira JA. Níveis de prática de atividade física habitual em adolescentes. Rev Bras Med Esporte 2001;7(6):187-199.

9. França AA, Kneube DPF, Souza-Kaneshima AM. Hábitos alimentares e estilo de vida de adolescentes estudantes na rede pública de ensino da cidade de Maringá/PR. Iniciação Científica CESUMAR 2006;8(2):175-183.

10. Sallis JF \& Owen N. Physical Activity \& Behavioral Medicine. Thousand Oaks: Sage Publications, 1999.
11. Almeida SS, Nascimento PCBD, Quaioti TCB. Quantidade e qualidade de produtos alimentícios anunciados na televisão brasileira. Rev Saúde Pública 2002;36(3):353-355.

12. Alves JGB. Atividade física em crianças: promovendo a saúde do adulto. Rev Bras Saúde Mater Infant 2003;3(1):5-6.

13. Matos M, Simões C, Canha L, Fonseca S. Saúde e estilo de vida nos jovens portugueses. Lisboa: FMH/ PPES; 2000.

14. Pires MC, Lopes AS. Crescimento físico e características sócio-demográgicas em escolares no município de Florianópolis - SC, Brasil. Rev Bras Cineantropom Desempenho Hum 2004;6(2):17-26

15. Oehlschlaeger MHK, Pinheiro RT, Horta B, Gelatti C, Sant'ana P. Prevalência e fatores associados ao sedentarismo em adolescentes da área urbana. Rev Saúde Pública 2004;38(2):157-163.

16. Ceschini FL. Nível de atividade em adolescentes de uma escola pública do Distrito da Vila Nova Cachoeirinha em São Paulo - SP. [Dissertação de Mestrado - Programa de Pós Graduação em Saúde Pública]. São Paulo (SP): Universidade de São Paulo; 2007.

17. Kazapi IM, Di Pietro PF, Avancini SRP, Freitas SFT, Tramonte VLCG. Consumo de energia e macronutrientes por adolescentes de escolas públicas e privadas. Rev Nutr 2001;14 (suppl. 0):27-33.

18. Santos JS, Costa COM, Nascimento Sobrinho CL, Silva MCM, Souza KEP, Melo BO. Perfil antropométrico e consumo alimentar de adolescentes de Teixeira de Freitas - Bahia. Rev Nutr 2005;18(5):623-632.

\section{Endereço para correspondência}

Eliane Denise da Silveira Araújo

Rua: João Cecy Filho, 175 Bairro: Uvaranas

CPE: 84020-020. Ponta Grossa, PR. Brasil

E-mail: eds_araujo@hotmail.com 